\title{
El Banco Asiático de Inversión en Infraestructura apuntando al camino del medio: uniéndose a las filas de los Bancos Multilaterales de Desarrollo, pero con características chinas
}

The Asian Infrastructure Investment Bank treading the middle path: joining the ranks of MDBs, but with Chinese Characteristics

\section{O Banco Asiático de Investimento em Infraestrutura apontando para o caminho do meio: unir as fileiras dos Bancos Multilaterais de Desenvolvimento, mas com características chinesas}

Andres Borquez ${ }^{1}$

Faran Naru ${ }^{2}$

DOI: 10.5752/P.2317-773X.2019v7.n3.p103

Recibido el 19 de noviembre de 2018

Aprobado el 13 de marzo de 2019

1. Investigador Instituto de Estudios Internacionales, Universidad de Chile, Santiago de Chile. Doctor en Políticas Internacionales, Universidad de Fudan, China https://orcid.org/0000-0002-3904409X (iD)

2. Profesor Asistente, Center for Public Policy and Governance, Forman Christian College, Pakistan. Doctor en Políticas Internacionales, Universidad de Fudan, China (iD)

\section{RESUMEN}

Durante 2016 fue aprobado el primer proyecto del Banco Asiático de Inversión en Infraestructura y una de las preocupaciones de los expertos es que esta nueva institución puede ser flexible con respecto a las condiciones de los préstamos y salvaguardas, argumentando que los creadores de este banco carecen de experiencia suficiente para mantener altos estándares establecidos por los otros bancos multilaterales. En contraste, otros especialistas destacan que la participación de China en el financiamiento a nivel internacional no es nada nuevo. Durante la última década, China ha permitido que los bancos de desarrollo estatales otorguen créditos no concesionales. Este artículo analiza ambos enfoques mediante un análisis comparativo del diseño de los bancos de desarrollo regionales y el nuevo banco multilateral liderado por China. Además, analiza la orientación de las políticas de crédito y salvaguardas de los 21 primeros proyectos aprobados por AIIB hasta el 2017. El nuevo banco esta apuntando al camino del medio: por un lado, uniéndose a las filas de los principales bancos multilaterales, pero al mismo tiempo, tratando de ser una institución con una visión sur a sur: ágil, respetando las políticas internas de cada país y enfocada en el nicho de los proyectos de infraestructura. 
Palabras claves: Banco Asiático de Inversión en Infraestructura (AIIB), Bancos del Desarrollo, Identidad de Instituciones Multilaterales, Políticas económicas exteriores de China.

\section{ABSTRACT}

In June 2016 the first project of the AIIB was approved and some experts' concern is if this new institution can be flexible with respect to the conditions of the loans and safeguards, arguing that the creators of this bank lack enough experience to maintain high standards established by the other multilateral banks. In contrast, other specialists point out that China's participation in financing at an international level is nothing new. Over the past decade, China has allowed state development banks to grant non-concessional loans. This article analyzes both approaches and contrasts them with a comparative analysis of the design of the regional development banks and the new multilateral bank led by China. In addition, it analyzes the orientation of the credit policies and safeguards of the first 21 projects approved by AIIB until 2017. The new bank is treading the middle path: on the one hand, joining the ranks of the main multilateral banks, but at the same time, trying to be an institution with a south-south vision: agile, respecting the internal policies of each country and focused on the niche of infrastructure projects.

Keywords: Asian Investment Bank in Infrastructure (AIIB), Development Banks, Identity of Multilateral Institutions, China’s Foreign Economic Policies

\section{RESUMO}

Em 2016, foi aprovado o primeiro projeto do Banco Asiático de Investimento em Infraestrutura (AIIB). Uma das preocupações de alguns especialistas é que essa nova instituição seja flexível com relação às condições de empréstimos e salvaguardas, ademais argumentam que os criadores desse banco não têm experiência suficiente para manter altos padrões estabelecidos pelos outros bancos multilaterais. Outros especialistas enfatizam que a participação da China no financiamento internacional não é novidade, salientando que, na última década, a China permitiu que os bancos estaduais de desenvolvimento realizassem empréstimos não concessionais. Este artigo analisa essas duas abordagens por meio de uma análise comparativa do design dos bancos de desenvolvimento regional e do novo banco multilateral liderado pela China. Além disso, analisa a orientação das políticas de crédito e salvaguardas dos 21 primeiros projetos aprovados pelo AIIB até 2017. O novo banco aponta para o caminho do meio: por um lado, ingressar nas fileiras dos principais bancos multilaterais e, ao mesmo tempo, tentar ser uma instituição com uma visão sul-sul: ágil, respeitando as políticas internas de cada país e focada no nicho de projetos de infraestrutura.

Palavras-chave: Banco Asiático de Investimento em Infraestrutura (AIIB), Bancos de Desenvolvimento, Identidade de Instituições Multilaterais, Políticas Econômicas Estrangeiras da China

Introducción: rompecabezas retórico.

Banco Asiático de Inversión en Infraestructura (AIIB en sus siglas en inglés) es un nuevo Banco Multilateral de Desarrollo (MDB) que tiene como objetivo satisfacer la demanda de financiamiento de infraestructura de Asia. Esta nueva institución intenta brindar una solución a la proyección del Banco Asiático de Desarrollo (ADB en sus siglas de inglés) en 
el 2009; donde sostenía que el continente asiático necesitaría 8 billones de dólares en los próximos 10 años, monto que no podría ser cubierto por el Banco Mundial (WB en sus siglas en inglés) y ADB (ADB, 2009). Desde la propuesta de AIIB en 2013, más de 64 países se han unido a este banco comprometiéndose a participar como fundadores o miembros no regionales. Entre estos países se encuentran las principales potencias europeas: Alemania y Reino Unido.

Esto simboliza un gran triunfo para Beijín, sin embargo, el éxito inicial no ha estado exento de resistencias. Estados Unidos y Japón, que son miembros fundadores y principales financiadores del Banco Mundial (WB) y del Banco Asiático de Desarrollo (ADB), han mostrado un entusiasmo limitado y se han restado de participar de esta nueva institución. Estos países han expresado sus dudas sobre si los préstamos de AIIB pueden cumplir con los criterios crediticios y los estándares ambientales y sociales. Adicionalmente, ambos países han criticado el rol de esta nueva institución como instrumento de política exterior de Beijín, basada en lograr objetivos estratégicos y económicos a costa de la región.

No es casualidad que los dos últimos bancos multilaterales estén respaldados por China. El gigante asiático se encuentra abandonado su rol como jugador secundario en el quehacer internacional y ha ido ingresando gradualmente a un dominio controlado por los bancos Bretton Woods, los cuales son caracterizados por el amplio respaldo de Estados Unidos. Después de la creación de New Development Bank (NBD también conocido como banco BRICS), y AIIB; China ha quebrado el monopolio del sistema de Bretton Woods durante 70 años. Esto ha generado una serie de reacciones en la comunidad internacional. En particular, la creación de AIIB, se considera como un fuerte competidor para ADB en términos de membresía y suscripción de capital. En este contexto, varios académicos y especialistas coinciden en que el establecimiento de estos nuevos bancos, principalmente el AIIB, redefinirán el sistema financiero global, lo cual ha conllevado a la generación de posturas retóricas contrapuestas.

Por un lado, se encuentra una visión integradora o complementaria: académicos como Gu (2017) y Ren (2016) argumentan que la creación de bancos multilaterales complementarios puede proporcionar apoyo financiero y técnico adicional en regiones con bajo nivel de desarrollo. En este sentido, se considera que AIIB es una buena oportunidad para resguardar áreas de impacto más amplias y cubrir una brecha de inversión necesaria en el desarrollo económico de Asia (HANLON, 2017). Shahar \& Lee (2018, p. 575) sostienen que este nuevo banco ha creado un contrapeso Sur-Sur para el Banco Mundial y el Fondo Monetario Internacional, no obstante, sostener que AIIB refleja una postura revisionista del gobierno chino se encuentra fuera de lugar. En la misma línea, Chin \& Helleiner (2008) y Cai \& Peter (2009) sostienen que la participación de China en proyectos de infraestructura de financiamiento y consultoría a nivel internacional no es un fenómeno nuevo. Durante la última década, el gobierno de la República Popular China ha permitido regularmente a los bancos nacionales proporcionar prestamos concesionales internacionales. Lo anterior, también se ha visto reflejado en el trabajo de Gransow \& Price (2018) donde han examinado 
la gestión del riesgo social del nuevo banco, afirmando que esta nueva institución tiene un alto grado de alineación con las políticas de salvaguardia social de los MDB preexistentes. Finalmente, la creación de AIIB se percibe como una posibilidad de acelerar el proceso de otorgamiento de préstamos y de reducir las altas restricciones que a menudo causan tensión cuando los bancos se involucran en aspectos de política interna (GRIFFITH-JONES, 2014).

Por otro lado, AIIB también ha suscitado una visión crítica, donde algunos especialistas han tomado posiciones más escépticas sobre el objetivo político de este nuevo banco. LaForgia (2017) introduce la discusión sobre las oportunidades de softpower que representa para China esta nueva institución, señalando que AIIB tiene el potencial de dar una nueva forma a la gobernanza global. La especialista sostiene que la nueva narrativa que Beijín ha dispuesto para esta institución reafirma este punto, estableciendo que detrás del eslogan "lean, clean and green" (delgado, limpio y verde) el banco busca proyectar las ideas de una institución con un carácter astuto basado en una fuerte capacidad de respuesta, una actitud de liderazgo responsable y utilizando parámetros de sostenibilidad inteligente. Otros autores que abordan esta institución como una fuente de influencia son Ikenberry \& Lim (2017) y Hooijmaaijers (2015), los cuales argumentan que AIIB es parte de la estrategia de China para crear nuevas instituciones con propósitos contrahegemónicos. Los autores sostienen que este nuevo banco busca aumentar la autoridad de Beijín dentro del orden internacional existente, basado en un juego de suma cero que intenta reducir la influencia del occidente y en especial Estados Unidos en la región asiática. En esta misma línea autores como Frost (2014) señalan que el AIIB no solo puede ayudar a China a expandir su esfera de influencia política internacional, sino que también puede usarse para aliviar los problemas internos de sobrecapacidad y crecimiento moderado. Otras visiones críticas se basan en la preocupación por los procesos internos de AIIB. Kawai (2015), por ejemplo, argumenta que AIIB podría desarrollar un enfoque más flexible para las decisiones de crédito, las prácticas bancarias y las salvaguardas ambientales o sociales, lo que aumentaría la financiación de proyectos más riesgosos. Este autor sostiene que los creadores de AIIB carecen de experiencia suficiente para mantener los altos estándares y niveles de transparencia establecidos por los bancos existentes. En el mismo sentido, Kawai afirma que AIIB carece de una visión clara de los objetivos a largo plazo, señalando que, a diferencia de los bancos multilaterales existentes, no identifica la reducción de la pobreza como un objetivo principal.

Aunque los Artículos del Acuerdo de AIIB muestran grandes similitudes estructurales con los bancos existentes (optando por la adopción de los mecanismos existentes para mejorar las posibilidades de su propio éxito), los estudios preliminares no han arrojado luces sobre cómo opera AIIB con respecto a aspectos como las políticas de crédito y la estructura de gobierno (HUMPHREY, 2015). Este artículo se centra en estos aspectos, analizando el papel del AIIB en un esquema multilateral. Específicamente, este estudio argumenta que el nuevo banco busca su propia forma de reconocimiento, utilizando la experiencia de las instituciones existentes y generando nuevas metodologías y políticas. AIIB apunta al 
camino del medio, adaptándose a los estándares de otros bancos multilaterales importantes, pero también utilizando el conocimiento del modelo de financiamiento estatal chino de proyectos de infraestructura en países en desarrollo.

Para abordar este análisis, el siguiente artículo primero discute la idea teórica de la construcción de identidad en organismos internacionales adaptando la teoría de identidad social de Tajfel \& Turner (1979). Posteriormente, se realizan tres análisis comparados: primero se revisa la estructura del AIIB comparada con la de los principales bancos multilaterales (BMD). Se procede a discutir las similitudes en la sección titulada "unirse a las filas de los BMD mediante el cumplimiento de estándares". La segunda comparación es sobre las tácticas operativas de AIIB y otros MDB. En esta comparación se identifican las principales diferencias, las cuales son discutidas en la sección titulada "características chinas". La tercera comparación es entre los préstamos desembolsados por AIIB comparados con las políticas de los bancos de Bretton Woods, para mostrar cómo Beijín se esfuerza por cumplir con los estándares vigentes, pero al mismo tiempo está adoptando tácticas operacionales que ha ido aprendiendo de los Banco de Desarrollo de China y el Banco de Exportaciones e Importaciones de China. En un esfuerzo por ajustarse a otros BMD e incorporar también sus propias características, China está tratando de equilibrar esta nueva institución recorriendo el camino medio y, al hacerlo, está creando una nueva identificación de préstamos de Sur a Sur. Esta nueva identidad se explica en la sección final titulada "encontrando el camino medio".

Revisión de la literatura: las instituciones multilaterales y el aumento de la interdependencia mundial

El aumento de la interdependencia global no se puede pensar de esta manera sin considerar la influencia de las instituciones multilaterales. A pesar del resurgimiento de algunas corrientes proteccionistas en los países occidentales, cada año hay una multiplicación de iniciativas que buscan sofisticar los sistemas de cooperación internacional. Los países han entendido que la cooperación a través de instituciones internacionales permite que se entregue un marco formal de seguridad, pero también sistematizar la cooperación bajo los principios del beneficio mutuo y reciprocidad.

Si bien, desde una perspectiva realista, la cooperación internacional se explica como resultado de una estrategia de equilibrio de poder (MORGENTHAU, [1948]/2005), existen otros enfoques que dimensionan a la cooperación con lógicas menos orientadas al establecimiento de alianzas contra un adversario, como son las instituciones multilaterales (BERGSTEN, 1976; KEOHANE, 1984, p. 7). Estos mecanismos no solo implican el establecimiento de una organización formal implementada por especialistas que buscan la creación de un entorno favorable para el intercambio de necesidades, sino que también son instancias vinculantes por las cuales los estados negocian y ajustan sus comportamientos creando una interdependencia basada en la reciprocidad. 
Una de las críticas más comunes sobre las posiciones a favor de la cooperación institucional como mecanismo de interdependencia económica, es su ingenua visión sobre el poder y el conflicto, aludiendo al hecho de que usan premisas demasiado optimistas (KEOHANE, 1984). En tal situación, es indiscutible que la cooperación basada en instituciones multilaterales, como otros tipos de interacciones entre países, conlleva una serie de dificultades constantes, como los conflictos de intereses y el dilema del prisionero. Del mismo modo, también es ampliamente aceptado que los acuerdos de instituciones multilaterales implican dificultades particulares, como los problemas de interpretación y la adaptación de cláusulas en un sistema internacional cambiante (YOUNG, 1986, p. 122).

Sin embargo, la evidencia ha demostrado, especialmente durante estas últimas décadas, que más allá de estas dificultades, las instituciones internacionales han ido evolucionando, haciéndose cada vez más complejas. En este contexto, las estrategias de cooperación basadas en iniciativas multilaterales se han ido sofisticando, integrando nuevas tecnologías, modelos dinámicos de estructuras de gobiernos y sistemas de intercambio de información. En este sentido, las instituciones internacionales son instrumentos mutables que requieren una mejora continua, basados en la interacción mutua permanente (HAGGARD; SIMMONS, 1987).

En tal configuración, se puede deducir que tanto el enfoque basado en alianzas de cooperación de seguridad como el basado en la generación de instituciones de cooperación interdependientes incluyen motivaciones mixtas, caracterizadas por una combinación de intereses complementarios y conflictivos. La diferencia entre estos enfoques es que el primero otorga mayor importancia a la estructura del sistema internacional como factor determinante en el comportamiento defensivo u ofensivo de los estados, para lo cual se hace hincapié, en su supervivencia y en la maximización de su poder a expensas de terceras naciones (MEARSHEIMER, 2001). Por el otro lado, el enfoque basado en la cooperación interdependiente sostiene que la estructura del sistema internacional establece limitaciones intrínsecas y cambiantes, que pueden reducirse mediante la negociación, el intercambio de información y la aplicación descentralizada de las instituciones entre las naciones (NYE; KEOHANE, 1989).

\section{Marco Teórico: producción de identidad de nuevas instituciones} multilaterales

Hay un aumento en el uso del concepto de identidad en el campo de las relaciones internacionales, especialmente durante los últimos veinte años, gracias al advenimiento de las teorías críticas y constructivistas (ASHIZAWA, 2008). Inicialmente, la atención se había centrado en la construcción del proceso de identidad nacional y su relación con el desarrollo de las políticas públicas (BLOOM, 2003). Más tarde, académicos se centraron en las interacciones entre las identidades nacionales de los países y las organizaciones internacionales (EYRE; SUCHMAN, 1996). Los especialistas han prestado atención a las interacciones entre las identidades de los estados y sus formas de cooperar o competir a nivel in- 
ternacional (GARTZKE; GLEDITSCH, 2006), sin embargo, todavía hay pocos estudios que abordan el proceso de producción de identidad de las nuevas organizaciones internacionales. En este contexto, este estudio articula dos enfoques aplicados para las relaciones internacionales basados en la teoría de identidad social de Tajfel \& Turner (1979): 1) producir una identidad similar para ajustarse a las instituciones existentes, basado en una actitud diligente y cumpliendo con las normas establecidas del juego y 2) producir un nuevo estatus que refleje la política exterior de la nación promotora, basado en la búsqueda de nichos distintivos que permitan impulsar normas alternativas.

El primer enfoque posiciona la producción de identidad como un factor prominente para buscar el reconocimiento del sistema establecido. Esta perspectiva posiciona el concepto de identidad como un elemento funcional en el proceso de generación de estatus en el esquema global. En este sentido, la construcción de la identidad equivale a la validación de otros actores. Esta perspectiva está relacionada con uno de los argumentos de este artículo, donde la apertura de un nuevo banco internacional está vinculada al desarrollo de una identidad que permite a otros bancos y países reconocer a esta nueva institución. Lo anterior, funciona como un primer paso en el proceso de legitimación internacional, donde el grupo da un sentido de identidad social, un sentido de pertenencia al mundo (TAJFEL \& TURNER, 1979). En el caso de AIIB, visualiza su identidad como una organización que se adapta a los estándares de otros bancos multilaterales, mientras que al mismo tiempo reorienta ciertos procedimientos específicos a sus propios objetivos.

El segundo enfoque se centra en cómo la identidad de una nueva organización internacional incorpora el carácter del estado precursor y su marco de acción en la política exterior. En esta línea, la identidad y el reconocimiento operan como factores que modelan el comportamiento de las nuevas potencias emergentes que enfrentan el dilema entre adaptarse a las instituciones internacionales compitiendo bajo las lógicas de una rivalidad geopolítica o promover modelos alternativos que ayuden a cumplir sus propios intereses nacionales, pero sin generar un entorno puramente competitivo. En este punto, Tajfel \& Turner (1979) plantean que los individuos pueden optar por una estrategia de carácter creativo, donde se incluyen las normas estructurales del sistema, pero abarcando nichos que permitan cambiar algunas referencias del grupo.

Para el caso de AIIB, Beijín ha optado por mantener una organización similar a la del grupo de bancos líderes en créditos para los países en desarrollo. No obstante, ha puesto el foco en especializarse en el nicho de proyectos de infraestructura y conectividad, exhibiendo algunas modificaciones operacionales que le permiten obtener influencia como un actor válido en la materia.

En esta configuración, promover instituciones alternativas como el AIIB, no responden acciones predeterminadas, sino que son el resultado de negociaciones entre actores nacionales e internacionales (MILNER; KEOHANE, 1996). En este punto, se debe enfatizar que cada decisión de preferencias responde a una evaluación de dos niveles, en la que los intereses nacionales e internacionales representan dos la- 
dos del mismo proceso de negociación que están conectados entre sí (PUTNAM, 1998). Este caso muestra cómo la postura de China hacia los bancos multilaterales se ha desarrollado, desde una perspectiva inicial de búsqueda de mayor protagonismo en las instituciones existentes, hasta la promoción de normas y reglas alternativas a través de nuevas instituciones financieras para los países en desarrollo. En esta producción, el nuevo banco busca su propio estatus, aprovechando la experiencia de las instituciones existentes y reorientando ciertas políticas y metodologías.

Uniéndose a las filas mediante el cumplimiento de estándares

Hace cuatro décadas, China llamó la atención del mundo, como un estado comunista que renunció al socialismo ortodoxo y comenzó una nueva etapa de reformas orientadas hacia la apertura del mercado. Ahora, China está de nuevo en la lupa internacional, pero esta vez como una nación que está ad-portas de convertirse en el nuevo líder del desarrollo económico asiático. En este esfuerzo, el AIIB es uno de los vehículos centrales de las políticas exteriores del gigante asiático. Esta nueva institución se presenta como un contrapeso a las instituciones de Bretton Woods, ofreciendo una nueva opción a los países en desarrollo para financiar sus proyectos de infraestructura. En este contexto, las autoridades de Beijín han desarrollado nuevas iniciativas multilaterales en las que buscan no solo compartir el riesgo-beneficio de las operaciones bancarias, sino también el conocimiento, incorporando estándares internacionales como parte de la estructura de estas nuevas instituciones. Esto queda claro al comparar las características principales de AIIB con los bancos multilaterales existentes, donde se puede observar similitudes estructurales y diferencias operacionales. A continuación, se procede abordar estos elementos:

Tabla 1 - Comparación entre los bancos regionales y AllB

\begin{tabular}{|c|c|c|c|c|c|}
\hline Factores Claves & AIIB & $\mathrm{ADB}$ & IDB & AfDB & EBRD \\
\hline Objetivo principal & $\begin{array}{l}\text { Desarrollo de Infraestruc- } \\
\text { tura }\end{array}$ & $\begin{array}{l}\text { Reducción de la po- } \\
\text { breza \& Desarrollo } \\
\text { Económico }\end{array}$ & $\begin{array}{l}\text { Reducción de la } \\
\text { pobreza \& Desarrollo } \\
\text { Económico }\end{array}$ & $\begin{array}{l}\text { Reducción de la po- } \\
\text { breza \& Desarrollo } \\
\text { Económico sustentable }\end{array}$ & $\begin{array}{l}\text { Desarrollo } \\
\text { Económico suste- } \\
\text { ntable }\end{array}$ \\
\hline Países Miembros & 64 & 67 & 48 & 64 & 80 \\
\hline Capital subscrito (USD) & 100 Billion & 163 Billion & 144 Billion & 80 Billion & 40 Billion \\
\hline Estructura de gobierno & \multicolumn{5}{|c|}{ a) Consejo de Gobernadores, b) Junta de Directiva, c) Administración } \\
\hline Evaluación de proyectos & \multicolumn{5}{|c|}{ a) Objetivos, b) impactos, c) políticas, d) fuente de financiamiento alternativo } \\
\hline $\begin{array}{l}\text { Ventanilla de financia- } \\
\text { miento }\end{array}$ & Sin Ventanilla concesional & Ventanilla concesional & $\begin{array}{l}\text { Ventanilla conce- } \\
\text { sional }\end{array}$ & Ventanilla concesional & $\begin{array}{l}\text { Ventanilla conce- } \\
\text { sional }\end{array}$ \\
\hline 5 mayores accionistas & $\begin{array}{l}\text { China, India, Rusia, Ale- } \\
\text { mania, Sur Corea }\end{array}$ & $\begin{array}{l}\text { Japón, EE. UU., China, } \\
\text { India, Australia }\end{array}$ & $\begin{array}{l}\text { EE. UU., Argentina, } \\
\text { Brasil, México, Japón }\end{array}$ & $\begin{array}{l}\text { EE. UU., Nigeria, } \\
\text { Japón, Egipto, África } \\
\text { del sur }\end{array}$ & $\begin{array}{l}\text { EE. UU., Francia, } \\
\text { Alemania, Italia, } \\
\text { Japón }\end{array}$ \\
\hline Derecho a voto regional & $75 \%$ & $65.1 \%$ & $50 \%$ & $59.5 \%$ & $13.8 \%$ \\
\hline Junta de directores & Sin residencia & Con residencia & Con residencia & Con residencia & Con residencia \\
\hline $\begin{array}{l}\text { Ratio capital desem- } \\
\text { bolsado }\end{array}$ & $20 \%$ & $5 \%$ & $3.7 \%$ & $4.9 \%$ & $20.9 \%$ \\
\hline
\end{tabular}

Nota: Compilación del autor en base a información de los sitios web oficiales. 
La estructura organizativa y de financiamiento de AIIB muestra similitud con los bancos multilaterales existentes.

En síntesis, el banco se divide en tres grandes áreas: Consejo de Gobernadores, Junta de Directores y el Equipo Administrativo del banco. El Consejo de Gobernadores consiste en las delegaciones de los países miembros. Por otro lado, las decisiones del día a día, como la aprobación de un proyecto de préstamo, son tomadas por la Junta Directiva. La gestión de AIIB está dirigida por el presidente y el vicepresidente. Estos elementos son semejantes a los otros bancos del desarrollo existentes, no obstante, la principal diferencia en términos de estructura organizativa es que el AIIB cuenta con una junta de directores no residentes. Esto se traduce en mayores responsabilidades operativas para la casa matriz y el presidente del banco. Algunos expertos como Tang argumentan que esto podría debilitar el papel de los accionistas (TANG, 2015). Los directores de la junta tienen funciones limitadas y no participan en las decisiones diarias del banco. En este sentido, los artículos de fundación del banco no mencionan el poder de los directores con respecto a la liberación o rechazo de un proyecto específico. Una explicación alternativa de esta política es que las autoridades de AIIB buscan implementar una estructura simple que no genere un exceso burocrático y que pueda acelerar el proceso de toma de decisiones.

En términos generales, el banco se financia con capital de los países miembros. AIIB emite bonos en los mercados internacionales, con el fin de recaudar nuevos fondos que luego se utilizan para préstamos a países emergentes. El único matiz es que AIIB no ofrece préstamos en base a financiamiento concesional. Esto se debe principalmente a que la misión de AIIB se enfoca solamente en proyectos de infraestructura regional, mientras que los objetivos de los otros bancos son la erradicación de la pobreza y el desarrollo social. En este sentido, AIIB no planea operar en condiciones concesionarias a corto plazo, puestas que las tasas se aplican para estos casos son a proyectos sociales con un impacto de largo plazo.

\section{AIIB cuenta con porcentajes de mayoría requeridos similares a los están-} dares de $\mathrm{MDB}$

En el caso de AIIB, las decisiones vinculantes requieren al menos una mayoría del 75\%, mismo porcentaje requerido en el ADB y en el Inter-American Development Bank (IDB). Sin embargo, se ha observado que la manera de lograr mayorías es diferente en AIIB. A pesar de la amplia participación internacional en el proceso de conformación del banco, el capital de suscripción y la participación en la votación corresponde principalmente a los países asiáticos. El 75\% de la suscripción de capital corresponde a países asiáticos, con una fuerte participación de China (29.8\% del capital total suscrito). La misma situación se aplica a la participación con derecho a voto, donde los países de la región asiática representan el $73.2 \%$ del total de votos, y dentro de eso China tiene la mayor participación con el $26.1 \%$ (ver tabla 2).

La participación asiática se vuelve altamente significativa cuando se considera que el $75 \%$ de los votos son necesarios para obtener la ma- 
yoría. En esta configuración, la asignación del poder de voto muestra un enfoque regionalista, donde el mayor porcentaje de votos es para China, seguido por India con un 7,5\% (véase el cuadro 2). Cabe señalar que la representación de China es casi igual a la suma del poder de voto de todos los países no regionales (26.7\%). Este punto tiene una alta incidencia en la búsqueda de mayorías para decisiones estratégicas, porque el porcentaje requerido para alcanzar mayorías es igual al porcentaje de acciones con derecho a voto de los países regionales. En otros bancos multilaterales, la búsqueda de las mayorías depende principalmente de la creación de consenso con accionistas no regionales (KAWAI, 2015).

Tabla 2 - Acciones de suscripciones de capital y poderes de voto (57 miembros fundadores)

\begin{tabular}{|c|c|c|c|}
\hline & Capital Subscrito (\$ Billones) & Capital (\%) & Poder de voto (\%) \\
\hline Regional & 75 & 75 & 73.2 \\
\hline China & 29.8 & 30.3 & 26.1 \\
\hline India & 8.4 & 8.5 & 7.5 \\
\hline Rusia & 6.5 & 6.7 & 5.9 \\
\hline Corea del sur & 3.7 & 3.8 & 3.5 \\
\hline Australia & 3.7 & 3.8 & 3.5 \\
\hline Indonesia & 3.4 & 3.4 & 3.2 \\
\hline Turquía & 2.6 & 2.7 & 2.5 \\
\hline Arabia Saudita & 2.5 & 2.6 & 2.5 \\
\hline Irán & 1.6 & 1.6 & 1.6 \\
\hline Tailandia & 1.4 & 1.5 & 1.5 \\
\hline Otros & 11.4 & 10.1 & 15.5 \\
\hline No-regionales & 25 & 25 & 26.7 \\
\hline Alemania & 4.5 & 4.6 & 4.2 \\
\hline Francia & 3.4 & 3.4 & 3.2 \\
\hline Brasil & 3.2 & 3.2 & 3 \\
\hline Reino Unido & 3.1 & 3.1 & 2.9 \\
\hline Italia & 2.6 & 2.6 & 2.5 \\
\hline España & 1.8 & 1.8 & 1.8 \\
\hline Holanda & 1 & 1.1 & 1.2 \\
\hline Polonia & 0.8 & 0.9 & 1 \\
\hline Suiza & 0.7 & 0.8 & 0.9 \\
\hline Suecia & 0.6 & 0.7 & 0.8 \\
\hline Otros & 3.3 & 2.8 & 5.2 \\
\hline
\end{tabular}

Fuente: Articulo del acuerdo AIIB (2015), GDP data IMF (2015), Center for Global Development (2015).

Características chinas

Experiencia de los bancos estatales del desarrollo chino

La República Popular de China (PRC) ha sido uno de los países del mundo que cambia más rápidamente. China ha experimentado importantes transformaciones políticas como: la búsqueda de la unidad después de la Guerra Civil; reformas sociales y agrarias; Revolución Cultural; la llegada del capitalismo a través del socialismo de mercado y la transfor- 
mación de China de un país pobre hacia una potencia mundial. Muchos de estos cambios fueron radicales, en particular considerando que se han seguido en poco tiempo (menos de 70 años) y que se han realizado bajo el mismo régimen político. En tal situación, es difícil clasificar la evolución de la política china en una teoría política clásica. Esta misma dificultad se aplica a la interpretación de su modelo económico y las lógicas detrás de sus relaciones internacionales.

El ascenso de China en la esfera internacional no solo puede entenderse desde una perspectiva económica, también se debe prestar atención a la actitud política del país internacionalmente y los mecanismos de vinculación utilizados. En este sentido, AIIB representa la búsqueda de aumentar la influencia global de China en la política internacional (zong he guo li). El gobierno liderado por el partido comunista de China ha perseguido el poder con estrategias que no necesariamente se ajustan a la definición perfecta del socialismo, capitalismo u otros ismos, por lo cual se identifica como las políticas internacionales con características chinas (FOOT, 2006).

En el caso del AIIB, es importante contextualizar que la participación de China en proyectos de financiamiento de infraestructura y consultoría a nivel internacional no es nada nuevo. Durante la última década, el gobierno de la República Popular China ha permitido que los bancos nacionales otorguen préstamos a las empresas chinas, teniendo acceso al uso del capital público a precios competitivos. En 2002, en el $16^{\circ}$ Congreso Nacional del Partido, Jiang Zemin alentó a las "empresas competitivas" a invertir en el exterior y "volverse global" (zou chu qu). Esta política se centró inicialmente en las adquisiciones estratégicas de activos de recursos naturales por parte de las empresas estatales como: CNPC, Sinopec y Chinalco, pero luego extendió su alcance al alentar a las empresas de múltiples sectores a invertir en el exterior. Los ejemplos incluyen la adquisición de la división de computadoras personales de IBM por Lenovo y la de Volvo por Geely Automobile. En esta estrategia, los bancos estatales chinos han desempeñado un papel activo en la autorización de préstamos con tasas de interés flexibles.

En términos operacionales, el sistema bancario chino incluye cuatro grandes bancos comerciales estatales, tres bancos del desarrollo (CDB, Banco ExIm y Banco de Desarrollo Agrícola de China) y un gran número de bancos comerciales más pequeños, cooperativas de crédito e instituciones financieras (ZHUANG; WHEALE, 2009, p. 112). No obstante, en relación con la financiación de proyectos de infraestructura en el exterior, los préstamos operan principalmente a través de dos instituciones estatales: China Development Bank (CDB) y China ExIm Bank (Chexim). Ambos bancos son financiados por emisiones de bonos en moneda china (Renminbi) y otros instrumentos financieros, comprados por bancos locales con la misma calificación crediticia que los bonos del gobierno chino (JIAJUN; CAREY, 2015). En escala y capacidades, CDB y China ExIm Bank se posicionan en el mismo nivel que los bancos multilaterales de desarrollo. Los bancos chinos de desarrollo utilizan tres enfoques de préstamos para estimular las inversiones en recursos naturales y proyectos de infraestructura: 1) ofrecer líneas de crédito a gobiernos extranje- 
ros y compañías de energía con préstamos garantizados con ingresos de ventas, generalmente a precios de mercado; 2) invertir en empresas chinas para la adquisición de recursos naturales a través de fondos de capital privado; y 3) apoyar a las empresas estatales chinas proporcionando financiamiento para las expansiones en el extranjero.

El conocimiento generado por los bancos chinos ha sido un importante apoyo para las nuevas instituciones multilaterales que lidera el gigante asiático a nivel internacional. Esto se refleja en el hecho de que la gran parte de la inversión directa de China en el extranjero (IED) de carácter no extractivo, se concentra en proyectos de infraestructura, transmisión energética y conectividad. Los últimos 15 años CDB ha proporcionado más de \$ 120 mil millones en líneas de crédito a las compañías chinas de telecomunicaciones, energía renovables y financiando programas de infraestructura (SANDERSON; FORSYTHE, 2012). Estas inversiones no solo han permitido al gigante asiático fortalecer su influencia a nivel internacional, sino que, en paralelo, posicionarse como una nación con alta capacidad de gestión de proyectos de infraestructura.

En este sentido, los bancos del desarrollo han adoptado una visión pragmática para los proyectos de infraestructura, dando prioridad a las iniciativas que representan un incentivo para el beneficio mutuo del país emisor y receptor (estrategia ganar-ganar). Con respecto a esto, los bancos han enfatizado el financiamiento de proyectos que son consistentes con la iniciativa de "La franja y la Ruta (Belt and Road Iniciative en inglés). Esta estrategia se concentra en fortalecer las relaciones interregionales y mejorar la conectividad física internacional. En la actualidad, China está involucrada en varios megaproyectos vinculados a esta iniciativa: el Canal de Nicaragua, el Proyecto de Agua del Norte del Sur de China y el Proyecto de la Cuenca del Río Mekong, entre otras. Estos proyectos abarcan una serie de obras tales como sistemas de transporte transnacionales, corredores bioceánicos, zonas económicas especiales y proyectos de conectividad digital.

\section{Principio de no interferencia}

Otra característica de los bancos del desarrollo chinos es la utilización del principio de no interferencia en las políticas internas de cada país. A pesar de que al gigante asiático le ha sido cada vez más complejo ser consistente con este principio, debido a su mayor compromiso con los organismos internacionales y su nuevo rol como líder responsable, esta actitud se ha mantenido desde el ascenso al poder del partido en el año 1949 (PAN; DU, 2015). A través de su visión de una sociedad armoniosa, las autoridades chinas se han impuesto recalcar la importancia de la no interferencia como principio rector en sus cooperaciones internacionales (ZHENG, 2016). Esto se ha visto reflejado sistemáticamente en los libros blancos de las políticas exteriores de Beijín, donde resaltan el mantenimiento del principio de desarrollo pacífico de China, basado en el respeto a que los países elijan independientemente su propio sistema económico-social y el camino al desarrollo (Ministerio de Relaciones Exteriores de China, 2016). Este punto no es menor, puesto que las autorida- 
des chinas han tratado de evitar lo que les sucedió a los bancos liderados por Estados Unidos (Banco Mundial y el Fondo Monetario Internacional) cuando fueron relacionados directamente con el consenso de Washington y el paquete de reformas estándar para los países en desarrollo que requiriesen los servicios de estas instituciones.

\section{Pólizas de salvaguardas estatales}

Finalmente, en relación con el nivel de salvaguardas, internamente los bancos del desarrollo chino utilizan su propia política llamada de "póliza de salvaguardas" de carácter estatal donde establecen las condiciones para las inversiones en el extranjero en nombre del gobierno chino. En términos generales, el nivel de requisitos es similar al de los bancos multilaterales existentes, excepto que prestan más atención al cumplimiento de las regulaciones ambientales del país receptor que a las regulaciones ambientales internacionales (ver la tabla 3). Por otro lado, los bancos chinos no tienen una política de reclamos y un monitoreo independiente, lo que hace difícil para los agentes externos hacer un seguimiento cuando se rompen las normas internacionales. Sin embargo, en la evaluación después de la realización de los proyectos, los bancos chinos tienen políticas de salvaguardas más estrictas que sus pares internacionales, centrándose en el seguimiento de largo plazo de los impactos.

En esta configuración, las autoridades chinas han establecido directrices complementarias, con el fin de proporcionar incentivos para el cumplimiento de las regulaciones ambientales y sociales. De esta manera, los agentes que actúan en paralelo con el banco han desarrollado nuevas salvaguardas por medio de directrices, como por ejemplo las creadas por la Comisión Reguladora Bancaria de China (directrices de crédito verde) y por el Ministerio de Comercio (directrices para la protección del medio ambiente en el extranjero).

En términos empíricos, estudios como el trabajo de Ray (2015) sobre proyectos financiados por bancos estatales chinos en América Latina muestran resultados mixtos: por un lado, varias compañías chinas han superado los estándares locales y han tenido un desempeño similar a los estándares internacionales, pero, por otro lado, hay proyectos cuya situación con respecto al cumplimiento de estándares son menos evidentes.

Tabla 3 - Regulaciones de bancos chinos y bancos multilaterales

\begin{tabular}{|l|c|c|c|}
\hline Indicadores & Estándar Bretton Wood & CDB & ExIm Bank \\
\hline Evaluación Impacto ambiental ex-ante & $\mathrm{X}$ & $\mathrm{X}$ & $\mathrm{X}$ \\
\hline Revisión de las evaluaciones de impacto ambiental & $\mathrm{X}$ & $\mathrm{X}$ & $\mathrm{X}$ \\
\hline Cumplimiento de la normativa ambiental del país anfitrión. & $\mathrm{X}$ & $\mathrm{X}$ & $\mathrm{X}$ \\
\hline Cumplimiento de la normativa ambiental internacional. & $\mathrm{X}$ & $\mathrm{X}$ \\
\hline Consultas públicas con potenciales comunidades afectadas. & $\mathrm{X}$ & \\
\hline Mecanismo de reclamo & $\mathrm{X}$ & & \\
\hline Monitoreo y revisión independiente & $\mathrm{X}$ & $\mathrm{X}$ & $\mathrm{X}$ \\
\hline Evaluaciones de Impacto ambiental ex-post & & $\mathrm{X}$ \\
\hline
\end{tabular}

Fuente: CBRC (2012), Ray et al (2015). 
Encontrando el camino del medio

El debate sobre la identidad de AIIB se centra en si este banco es el resultado de la cooperación financiera internacional o una herramienta china para promover sus intereses. Este último argumento se considera una explicación detrás de la negativa de Estados Unidos y Japón a unirse a esta nueva institución.

Estados Unidos, ha sido pionero en el desarrollo de instituciones financieras internacionales, a su vez, Japón, ha monopolizado el mercado financiero asiático, a través de su contribución con el Banco Asiático de Desarrollo y su influencia resultante del mismo. En este sentido, surge la interrogante ¿Es cuestionable la identidad del AIIB porque China, a través de este banco, ocupa territorio que antes era exclusivo de Estados Unidos o Japón? La respuesta es claramente no.

Quince de los veintiún proyectos aprobados por AIIB, a partir de abril del 2017, se cofinancian con el WB y ADB, entre otros prestadores (ver tabla 4). Del mismo modo, se puede observar que el AIIB ha decidido utilizar en la gran parte de los proyectos cofinanciados las mismas políticas de salvaguardia ambiental y social que el WB y el ADB. Quince de veintiún proyectos aprobados siguen las normas ambientales y laborales establecidas por el WB o ADB (ver tabla 4).

Las críticas hacia el AIIB no están surgiendo debido a su penetración en el mercado que antes era un negocio de préstamos "Norte a Sur" liderado por países desarrollados, sino se deben a la fuerza política económica detrás de este nuevo banco: China.

Las presiones internacionales que intentan contener la expansión económica de China internacionalmente inciden en el posicionamiento de la identidad del AIIB debido a la afiliación inseparable del país. Estados Unidos y Japón, entre otras potencias han tensionado el estatus de economía de no mercado de China en la OMC para restringir sus exportaciones a través de derechos antidumping (RUI PAN, 2015). Del mismo modo, se han planteado reiteradas preocupaciones medioambientales por las inversiones financiadas por los bancos de desarrollo chinos (GALLAGHER, 2013). Dichas restricciones y críticas apuntan a limitar el alcance económico global de la China basado en el crecimiento. Si bien, un país puede ser objeto de una política internacional de este tipo, un banco internacional que pretende fortalecer la inversión en proyectos de infraestructuras de los países asiáticos en desarrollo no debe convertirse en el objetivo de tales maniobras.

En tal configuración, China más allá de albergar la sede y ser el principal contribuyente de los fondos de esta nueva institución, busca coparticipar en las decisiones de los nuevos proyectos de infraestructura regional, compartiendo los potenciales riesgos y beneficios con otros países e instituciones. Los primeros proyectos aprobados reflejan esta actitud, ubicando al banco al medio del camino, siguiendo las lógicas de los bancos existentes, pero incorporando algunas características chinas que resaltan su carácter pragmático. 
Tabla 4 - Orientación de los primeros proyectos aprobados por AllB (2016-2017)

\begin{tabular}{|c|c|c|c|c|}
\hline Proyecto & País & Sector & Orientación crediticia & Orientación salvaguardas \\
\hline 1) Andhra Pradesh & India & Energía & $\begin{array}{l}\text { cofinanciado con Banco } \\
\text { Mundial (WB) }\end{array}$ & $\begin{array}{l}\text { Salvaguardas ambientales y socia- } \\
\text { les del WB (ESSP, siglas en inglés) }\end{array}$ \\
\hline $\begin{array}{l}\text { 2) Natural Gas Infrastructure \& Efficiency } \\
\text { Improvement }\end{array}$ & Bangladesh & Energía & $\begin{array}{l}\text { cofinanciado con Banco } \\
\text { Asiático de desarrollo (ADB) }\end{array}$ & $\begin{array}{l}\text { Salvaguardas del ADB (SPS, siglas } \\
\text { en inglés) }\end{array}$ \\
\hline $\begin{array}{l}\text { 3) Dam Operational Improvement and } \\
\text { Safety II }\end{array}$ & Indonesia & Multi-sector & cofinanciado con BM & Salvaguardas WB (ESSP) \\
\hline $\begin{array}{l}\text { 4) Regional Infrastructure Development } \\
\text { Fund }\end{array}$ & Indonesia & Multi-sector & cofinanciado con BM & Salvaguardas WB (ESSP) \\
\hline 5) Trans Anatolian Natural Gas Pipeline & Azerbaijan & Energía & cofinanciado con BM & Salvaguardas WB (ESSP) \\
\hline 6) Sultanate of Oman & Oman & Transporte & Financiado por AIIB & $\begin{array}{l}\text { Salvaguardas ambientales y socia- } \\
\text { les del AIIB (ESP, siglas en inglés) }\end{array}$ \\
\hline 7) Sultanate of Oman Railway System & Oman & Transporte & Financiado por AIIB & Salvaguardas AIIB (ESP) \\
\hline 8) Tarbela 5 Hydropower Extension & Paquistán & Energía & cofinanciado con BM & Salvaguardas WB (ESSP) \\
\hline 9) Myingyan 225 MW Power Plant & Myanmar & Energía & cofinanciado con BAD & Salvaguardas AIIB (ESP) \\
\hline 10) National Slum Upgrading & Indonesia & Multi-sector & cofinanciado con BM & Salvaguardas WB (ESSP) \\
\hline 11) Distribution System Upgrade \& Expansion & Bangladesh & Energía & Financiado por AIIB & Salvaguardas AIIB (ESP) \\
\hline 12) National Motorway M-4 & Paquistán & Transporte & cofinanciado con BAD & Salvaguardas ADB (SPS) \\
\hline 13) Nurek Hydropower Rehabilitation Project & Tajikistan & Energía & cofinanciado con BM & Salvaguardas WB (ESSP) \\
\hline 14) Gujarat Rural Roads (MMGSY) Project & India & Transporte & Financiado por AIIB & Salvaguardas AIIB (ESP) \\
\hline 15) Batumi Bypass Road Project & Georgia & Transporte & cofinanciado con BAD & Salvaguardas ADB (SPS) \\
\hline 16) Transmission System Strengthening Project & India & Energía & cofinanciado con BAD & Salvaguardas ADB (SPS) \\
\hline 17) Metro Manila Flood Management Project & Filipinas & Tratamiento Agua & cofinanciado con BM & Salvaguarda WB (ESSP) \\
\hline 18) Bangalore Metro Rail Project & India & Transporte & $\begin{array}{l}\text { cofinanciado con Banco Eu- } \\
\text { ropeo de inversión (EIB) }\end{array}$ & $\begin{array}{l}\text { Principios \& Estándares ambien- } \\
\text { tales EIB }\end{array}$ \\
\hline 19) Oman Broadband Infrastructure Project & Omán & Telecomunicaciones & Financiado por AIIB & Salvaguardas AIIB (ESP) \\
\hline $\begin{array}{l}\text { 20) Beijing Air Quality Improvement \& Coal } \\
\text { Replacement Project }\end{array}$ & China & Energía & Financiado por AIIB & Salvaguardas AIIB (ESP) \\
\hline $\begin{array}{l}\text { 21) Dushanbe-Uzbekistan Border Road } \\
\text { Improvement }\end{array}$ & Tajikistan & Transporte & $\begin{array}{l}\text { cofinanciado con Banco Eu- } \\
\text { ropeo para la reconstrucción } \\
\text { \& desarrollo (EBRD) }\end{array}$ & $\begin{array}{l}\text { Salvaguardas ambientales y } \\
\text { sociales EBRD }\end{array}$ \\
\hline
\end{tabular}

Nota: Compilación del autor basada en documentos oficiales publicados por el banco.

Conclusión

China ha posicionado a AIIB como una de sus prioridades en la nueva forma de liderazgo internacional que ha ido asumiendo durante los últimos años. Esta nueva institución se presenta como un contrapeso político de las instituciones de Bretton Woods, ofreciendo una nueva opción a los países en desarrollo para la financiación de sus proyectos de infraestructura. AIIB, junto con la Franja y la Ruta, son las iniciativas de softpower que más repercusión han tenido en la comunidad internacional. En esta configuración, China se ha proyectado como un país que busca aunar fuerzas en vías de una mayor interconectividad y apertura global, y así hacer frente a los nuevos vientos proteccionistas que comienzan a tomar fuerzas en las potencias occidentales.

Para muchos analistas, el buen funcionamiento de este nuevo banco garantiza al gigante asiático, una mayor influencia regional y un mayor poder en la gobernanza global. En este contexto, la construcción de 
la identidad y estatus de este banco desempeña un papel funcional en su proceso de reconocimiento a nivel internacional. En términos estructurales, tal como se discute en el marco teórico con la adaptación de la teoría de identidad social y en el apartado "uniéndose a las filas mediante el cumplimiento de estándares", AIIB ha tomado medidas significativas para adaptarse a los estándares de los bancos predecesores. Esto le permite reducir la incertidumbre en cuanto al funcionamiento general de la nueva institución y generar una validación inicial de los otros actores. No obstante, como se observa en el apartado "características chinas", el nuevo banco asiático intenta promover sus propias innovaciones especializándose solamente en proyectos de infraestructura y optando por instaurar nuevos mecanismos operacionales como son la concentración regional del poder de voto, los préstamos no concesionales, los directores no residentes y la incorporación del conocimiento práctico de los bancos de desarrollo chinos. Esto le permite posicionarse en un nicho específico evitando estimular un ambiente de híper competencia. Finalmente, como se observa durante los primeros tres años de funcionamiento, AIIB ha "encontrado el camino del medio", eligiendo préstamos moderados y prácticas socio ambientales estandarizadas. Esto le ha permitido obtener una alta calificación crediticia y reconocimiento, pero, al mismo tiempo, ha seguido insistiendo con crear un banco ágil, evitando un exceso de burocracia como parte de la nueva identidad del banco de cooperación sur-sur.

Referencias

ASHIZAWA, K. When Identity Matters: State Identity, Regional Institution-Building, and Japanese Foreign Policy. International Studies Review v.10, p. 571-598. 2008.

__ASIAN DEVELOPMENT BANK (ADB). Infrastructure for a Seamless Asia. Tokyo: Asian Development Bank Report. 2009. Disponible en: https://www.adb.org/sites/default/files/publication/159348/adbi-infrastructure-seamless-asia.pdf Visitado 12 Deciembre 2017.

BERGSTEN, F. Interdependence and the reform of international institutions. International organization v.30, n.2, p. 361-372. 1976.

BLOOM, W. Personal Identity, National Identity and International Relations. Cambridge: Cambridge University Press. 1993

CAI, Z. \& PETER W. Managing Efficient Capital Allocation with Emphasis on the Chinese Experience. Journal of Business Ethics, v.87, n.1, p. 111-135. 2009.

CHIN, G. \& HELLEINER, E. China as a creditor: a rising financial power? Journal of International affairs, v. 62, n.1, p.87-102. 2008.

CHINA BANKING REGULATORY COMMISION (CBRC). China banking regulatory commision annual report. 2012. Disponible en: http://www.cbrc.gov.cn/chinese/files/2013/4CF24B3E79704CEA85D330A7CC18CD7D.pdf Visitado 12 enero 2018.

EYRE D. \& SUCHMAN, M. Status, Norms and the Proliferation of Conventional Weapons: An Institutional Theory Approach. In the Culture of National Security: Norms and Identity in World Politics. New York: Columbia University Press. 1996.

FOOT, R. Chinese Strategies in a US-Hegemonic Global Order: Accommodating and Hedging. International Affairs, v.82, n.1, p.77-94. 2006.

FROST, E. Rival regionalisms and regional order. A slow crisis oflegitimacy. The national Bureau of Asian Research report n. 48, p. 1-30. 2014. http://www.nbr.org/publications/specialreport/pdf/ sr48_regional_architecture_december2014.pdf Visitado 11 diciembre 2017.

GALLAGHER, K. Profiting from Precaution: How China's Policy Banks Can Enhance Social and Environmental Standards. Chicago: Paulson Institute. 2013. 
GARTZKE, E. \& GLEDITSCH, K. Identity and Conflict: Ties That Bind and Differences That Divide. European Journal of International Relations v.12, n.1, p.53-87. 2006.

GRANSOW, B. \& PRICE, S. Social Risk Management at AIIB - Chinese or International Characteristics? Journal of Chinese Political Science, July, p. 1-23. 2018.

GRIFFITH-JONES, S. A BRICS development Bank: a dream coming true? United Nations Conference on trade and development Discussion Paper 21: 1-28. 2014. Disponible en: http://unctad.org/en/PublicationsLibrary/osgdp20141_en.pdf Visitado 12 enero 2018.

GU, B. Chinese Multilateralism in the AIIB. Journal of International Economic Law, v.20, p. 137158. 2017.

HAGGARD, S. \& SIMMONS, B. Theories of international regimes. International Organization, v.41, n.3, p. 491- 517. 1987.

HANLON, R. Thinking about the Asian Infrastructure Investment Bank: Can a China-Led Development Bank Improve Sustainability in Asia? Asia \& the Pacific Policy Studies, v.4, n.3, p.541554. 2017.

HOOIJMAAIJERS, B. The Asian Infrastructure Investment Bank: another wakeup call for the EU? Global Affairs, v.1, n.3, p. 325-334. 2015

HUMPHREY, C. Developmental revolution or Bretton Woods revisted? The prospect of the BRICS New Development Bank and the Asian Infrastructure Investment Bank. Shaping policy for development, working paper 418: 1-42. 2015. Disponible en: https://www.odi.org/sites/odi. org.uk/files/odi-assets/publications-opinion-files/9615.pdf Visitado 15 Noviembre 2017.

IKENBERRY, J. \& LIM, D. China’s emerging institutional statecraft: The Asian Infrastructure Investment Bank and the prospects for counter-hegemony. Brookings (April). 2017. Disponible en: https://www.brookings.edu/wp-content/uploads/2017/04/chinas-emerging-institutional-statecraft.pdf Visitado18 Noviembre 2017.

JIAJUN, X. \& CAREY, R. China's international development finance Past, present, and future. The World Institute for Development Economics Research Working paper 130. 2015. Disponible en: https://www.wider.unu.edu/sites/default/files/WP2015-130-.pdf Visitado 15 Febrero 2018.

KAWAI, M. Asian Infrastructure Investment Bank in the Evolving International Financial Order, In Asian Infrastructure Investment Bank: China as Responsible Stakeholder? Washington DC: Sasakawa Peace Foundation USA. 2015. Disponible en: https://spfusa.org/wp-content/ uploads/2015/07/AIIB-Report_4web.pdf Visitado 12 Dciembre 2017.

KEOHANE, R. After Hegemony: Cooperation and Discord in the world political economy. Princeton: University press (1st ed.). 1984.

LAFORGIA, R. Listening to China's multilateral voice for the first time: analysing the Asian infrastructure investment bank for soft power opportunities and risks in the narrative of 'lean, clean and green' Journal of Contemporary China v. 26, n.107, p. 633-649. 2017.

MEARSHEIMER, J. The Tragedy of Great Power Politics. New York: W. W. Norton \& Company. 2001.

MILNER, H. \& KEOHANE, R. Internationalization and domestic politics: an introduction. In Milner, Helen \& Keohane, Robert (eds.). Internationalization and domestic politics. United Kingdom: Cambridge University Press. 1996.

MINISTERIO DE RELACIONES EXTERIORES DE CHINA. Documento sobre la Política de China hacia América Latina y el Caribe. 2016. Disponible en: https://www.fmprc.gov.cn/esp/ wjdt/wjzc/t1418256.shtml visitado en 13 mayo 2018

MORGENTHAU, H J. Politics Among Nations: The Struggle for Power and Peace. New York: McGraw-Hill Education(7ed). [1948] 2005.

NYE, J. \& KEOHANE, R. Power and Interdependence: World Politics in Transition. New York: Little, Brown and Company. 1989.

PAN, Z. \& DU, P. The Logic of Contingency in China's Insistence on the Non-interference Principle, Fudan Journal Humanities and Social Sciences, v. 8, p. 597-615. 2015.

PUTNAM, R. Diplomacy and Domestic Politics: The Logic of Two-Level Games. International Organization v.42, p. 427-460. 1998.

RAY et al. China in Latin-American: lesson for south-south Cooperation and sustainable development. Boston: Global economic governance initiative report. 2015. Disponible en: https://www.bu.edu/pardeeschool/files/2014/12/Working-Group-Final-Report.pdf Visitado 15 Mayo 2018. 
REN, X. China as an institution-builder: the case of the AIIB. The Pacific Review, v.29, n.3, p. 435-442. 2016

SANDERSON, H. \& FORSYTHE, M. China's Superbank: Debt, Oil and Influence - How China Development Bank is Rewriting the Rules of Finance. New York: Bloomberg Press. 2012.

SHAHAR, H. \& LEE, J. China challenges global governance? Chinese international development finance and the AIIB, International Affairs, v. 94, n.3, p. 573-593. 2018.

TAJFEL, H. \& TURNER, J. Social comparison and group interest in ingroup favoritism. European Journal of Social Psychology, v. 9, 2, p. 187-204. 1979.

TANG, S. The Politics of the Asian Infrastructure Investment Bank (AIIB). Singapore: Institute of Southeast Asian Studies. 2015.

YOUNG, O. International Regimes: Toward a New Theory of Institutions. World Politics v. 39, p. 104-122. 1986.

ZHENG, C. China debates the Non-interference principle. The Chinese Journal of International Politics v. 9, n.3, p.349-374. 2016.

ZHUANG, C. \& WHEALE, P. Managing Efficient Capital Allocation with Emphasis on the Chinese Experience. Journal of Business Ethics v. 87, n.1, p. 111-135. 2009. 